\title{
Flow analysis, linearity, and PTIME
}

\author{
David Van Horn and Harry G. Mairson \\ Department of Computer Science \\ Brandeis University \\ Waltham, Massachusetts 02454 \\ \{dvanhorn, mairson\}@cs.brandeis.edu
}

\begin{abstract}
Flow analysis is a ubiquitous and much-studied component of compiler technology - and its variations abound. Amongst the most well known is Shivers' 0CFA; however, the best known algorithm for 0CFA requires time cubic in the size of the analyzed program and is unlikely to be improved. Consequently, several analyses have been designed to approximate 0CFA by trading precision for faster computation. Henglein's simple closure analysis, for example, forfeits the notion of directionality in flows and enjoys an "almost linear" time algorithm. But in making trade-offs between precision and complexity, what has been given up and what has been gained? Where do these analyses differ and where do they coincide?

We identify a core language - the linear $\lambda$-calculus - where 0CFA, simple closure analysis, and many other known approximations or restrictions to 0CFA are rendered identical. Moreover, for this core language, analysis corresponds with (instrumented) evaluation. Because analysis faithfully captures evaluation, and because the linear $\lambda$-calculus is complete for PTIME, we derive PTIME-completeness results for all of these analyses.
\end{abstract}

\section{Introduction}

Flow analysis [1-4] is concerned with providing sound approximations to the question of "does a given value flow into a given program point during evaluation?" The most approximate analysis will always answer yes, which takes no resources to compute - and is of little use. On the other hand, the most precise analysis will answer yes if and only if the given value flows into the program point during evaluation, which is useful, albeit uncomputable. In mediating between these extremes, every static analysis necessarily gives up valuable information for the sake of computing an answer within bounded resources. Designing a static analyzer, therefore, requires making trade-offs between precision and complexity. But what exactly is the analytic relationship between forfeited information and resource usage for any given design decision? In other words:

What are the computationally potent ingredients in a static analysis?

The best known algorithms to compute Shivers' 0CFA [5], a canonical flow analysis for higher-order programs, are cubic in the size of the program, and 
there is strong evidence to suggest that in general, this cannot be improved [6]. Nonetheless, information can be given up in the service of quickly computing a necessarily less precise analysis, avoiding the "cubic bottleneck." For example, by forfeiting 0CFA's notion of directionality, algorithms for Henglein's simple closure analysis run in near linear time [7]. Similarly, by explicitly bounding the number of passes the analyzer is allowed over the program, as in Ashley and Dybvig's sub-0CFA [8], we can recover running times that are linear in the size of the program. But the question remains: Can we do better? For example, is it possible to compute these less precise analyses in logarithmic space? We show that without profound combinatorial breakthroughs (PTIME = LOGSPACE), the answer is no. Simple closure analysis, sub-0CFA, and other analyses that approximate or restrict 0CFA, require - and are therefore, like 0CFA [9], complete for-polynomial time.

What is flow analysis? Flow analysis is the ubiquitous static analysis of higherorder programs. As Heintze and McAllester remark, some form of flow analysis is used in most forms of analysis for higher-order languages [10]. It answers fundamental questions such as what values can a given subexpression possibly evaluate to at run-time? Each subexpression is identified with a unique superscripted label $\ell$, which serves to index that program point. The result of a flow analysis is a cache $\widehat{C}$ that maps program points (and variables) to sets of values. These analyses are conservative in the following sense: if $v$ is in $\widehat{\mathrm{C}}(\ell)$, then the subexpression label $\ell$ may evaluate to $v$ when the program is run (likewise, if $v$ is in $\widehat{\mathrm{C}}(x), x$ may be bound to $v$ when the program is run). But if $v$ is not in $\widehat{\mathrm{C}}(\ell)$, we know that $e$ cannot evaluate to $v$ and conversely if $e$ evaluates to $v, v$ must be in $\widehat{\mathrm{C}}(\ell)$.

For the purposes of this paper and all of the analyses considered herein, values are (possibly open) $\lambda$-abstractions. During evaluation, functional values are denoted with closures - a $\lambda$-abstraction together with an environment that closes it. Values considered in the analysis approximate run-time denotations in the sense that if a subexpression labeled $\ell$ evaluates to the closure $\langle\lambda x . e, \rho\rangle$, then $\lambda x . e$ is in $\widehat{\mathrm{C}}(\ell)$.

The acceptability of a flow analysis is often specified as a set of (in)equations on program fragments. The most naive way to compute a satisfying analysis is to initialize the cache with the flow sets being empty. Successive passes are then made over the program, monotonically updating the cache as needed, until the least fixed point is reached. The more approximate the analysis, the faster this algorithm converges on a fixed point. The key to a fruitful analysis, then, is "to accelerate the analysis without losing too much information" [8].

One way to realize the computational potency of a static analysis is to subvert this loss of information, making the analysis an exact computational tool. Proving lower bounds on the expressiveness of an analysis becomes an exercise in hacking, armed with this newfound tool. Clearly the more approximate the analysis, the less there is to work with, computationally speaking, and the more we have to do to undermine the approximation. But a fundamental technique al- 
lows us to understand expressivity in static analysis: linearity. This paper serves to demonstrate that linearity renders a number of the most approximate flow analyses both equivalent and exact.

Linearity and approximation in static analysis: Linearity, the Curry-Howard programming counterpart of linear logic [11], plays an important role in understanding static analyses. The reason is straightforward: because static analysis has to be tractable, it typically approximates normalization, instead of simulating it, because running the program may take too long. For example, in the analysis of simple types - surely a kind of static analysis - the approximation is that all occurrences of a bound variable must have the same type (as a consequence, perfectly good programs are rejected). A comparable but not identical thing happens in the case of type inference for ML and bounded-rank intersection types - but note that when the program is linear, there is no approximation, and type inference becomes evaluation under another name.

In the case of flow analysis, similarly, a cache is computed in the course of an approximate evaluation, which is only an approximation because each evaluation of an abstraction body causes the same piece of the cache to be (monotonically) updated. Again, if the term is linear, then there is only one evaluation of the abstraction body, and the flow analysis becomes synonymous with normalization.

Organization of this paper: The next section introduces 0CFA in order to provide intuitions and a point of reference for comparisons with subsequent analyses. Section 3 specifies and provides an algorithm for computing Henglein's simple closure analysis. Section 4 develops a correspondence between evaluation and analysis for linear programs. We show that when the program is linear, normalization and analysis are synonymous. As a consequence the normal form of a program can be read back from the analysis. We then show in Section 5 how to simulate circuits, the canonical PTIME-hard problem, using linear terms. This establishes the PTIME-hardness of the analysis. Finally, we discuss other monovariant flow analyses and sketch why these analyses remain complete for PTIME and provide conclusions and perspective.

\section{Shivers' 0CFA}

As a starting point, we consider Shivers' 0CFA $[5,3] .{ }^{1}$

The Language: A countably infinite set of labels, which include variable names, is assumed. The syntax of the language is given by the following grammar:

$$
\begin{array}{lll}
\text { Exp } & e::=t^{\ell} & \text { expressions (or labeled terms) } \\
\text { Term } & t::=x|e e| \lambda x . e & \text { terms (or unlabeled expressions) }
\end{array}
$$

\footnotetext{
${ }^{1}$ It should be noted that Shivers' original zeroth-order control-flow analysis (0CFA) was developed for a core CPS-Scheme language, whereas the analysis considered here is in direct-style. Sestoft independently developed a similar flow analysis in his work on globalization $[2,12]$. See $[4,13]$ for details.
} 
All of the syntactic categories are implicitly understood to be restricted to the finite set of terms, labels, variables, etc. that occur in the program of interest the program being analyzed. The set of labels, which includes variable names, in a program fragment is denoted $\mathbf{l a b}(e)$. As a convention, programs are assumed to have distinct bound variable names.

The result of 0CFA is an abstract cache that maps each program point (i.e., label) to a set of $\lambda$-abstractions which potentially flow into this program point at run-time:

$$
\widehat{\mathrm{C}} \in \mathbf{L a b} \rightarrow \mathcal{P}(\text { Term }) \quad \text { abstract caches }
$$

Caches are extended using the notation $\widehat{\mathrm{C}}[\ell \mapsto s]$, and we write $\widehat{\mathrm{C}}\left[\ell \mapsto^{+} s\right]$ to mean $\widehat{C}[\ell \mapsto(s \cup \widehat{C}(\ell))]$. It is convenient to sometimes think of caches as mutable tables (as we do in the algorithm below), so we abuse syntax, letting this notation mean both functional extension and destructive update. It should be clear from context which is implied.

The Analysis: We present the specification of the analysis here in the style of Nielson et al. [14]. Each subexpression is identified with a unique superscripted label $\ell$, which marks that program point; $\widehat{\mathrm{C}}(\ell)$ stores all possible values flowing to point $\ell$. An acceptable flow analysis for an expression $e$ is written $\widehat{\mathrm{C}}=e$ :

$$
\begin{aligned}
& \widehat{\mathrm{C}} \models x^{\ell} \text { iff } \widehat{\mathrm{C}}(x) \subseteq \widehat{\mathrm{C}}(\ell) \\
& \widehat{\mathrm{C}} \models(\lambda x \cdot e)^{\ell} \text { iff } \lambda x \cdot e \in \widehat{\mathrm{C}}(\ell) \\
& \widehat{\mathrm{C}} \models\left(t_{1}^{\ell_{1}} t_{2}^{\ell_{2}}\right)^{\ell} \text { iff } \widehat{\mathrm{C}}=t_{1}^{\ell_{1}} \wedge \widehat{\mathrm{C}}=t_{2}^{\ell_{2}} \wedge \forall \lambda x \cdot t_{0}^{\ell_{0}} \in \widehat{\mathrm{C}}\left(\ell_{1}\right): \\
& \quad \widehat{\mathrm{C}} \models t_{0}^{\ell_{0}} \wedge \widehat{\mathrm{C}}\left(\ell_{2}\right) \subseteq \widehat{\mathrm{C}}(x) \wedge \widehat{\mathrm{C}}\left(\ell_{0}\right) \subseteq \widehat{\mathrm{C}}(\ell)
\end{aligned}
$$

The $\models$ relation needs to be coinductively defined since verifying a judgment $\widehat{\mathrm{C}} \equiv e$ may obligate verification of $\widehat{\mathrm{C}}=e^{\prime}$ which in turn may require verification of $\widehat{C}=e$. The above specification of acceptability, when read as a table, defines a functional, which is monotonic, has a fixed point, and $\models$ is defined coinductively as the greatest fixed point of this functional. ${ }^{2}$

Writing $\widehat{C} \models t^{\ell}$ means "the abstract cache $\widehat{C}$ contains all the flow information for program fragment $t$ at program point $\ell$." The goal is to determine the least cache solving these constraints to obtain the most precise analysis. Caches are partially ordered with respect to the program of interest:

$$
\widehat{\mathrm{C}} \sqsubseteq \widehat{\mathrm{C}}^{\prime} \text { iff } \forall \ell: \widehat{\mathrm{C}}(\ell) \subseteq \widehat{\mathrm{C}}^{\prime}(\ell) .
$$

Since we are concerned only with the least cache (the most precise analysis) we refer to this as the cache, or synonymously, the analysis.

The Algorithm: These constraints can be thought of as an abstract evaluator$\widehat{\mathrm{C}}=t^{\ell}$ simply means evaluate $t^{\ell}$, which serves only to update an (initially empty) cache.

\footnotetext{
${ }^{2}$ See [14] for a thorough discussion of coinduction in specifying static analyses.
} 


$$
\begin{aligned}
\mathcal{A} \llbracket x^{\ell} \rrbracket & =\widehat{\mathrm{C}}\left[\ell \mapsto^{+} \widehat{\mathrm{C}}(x)\right] \\
\mathcal{A} \llbracket(\lambda x . e)^{\ell} \rrbracket= & \widehat{\mathrm{C}}[\ell \mapsto\{\lambda x . e\}] \\
\mathcal{A} \llbracket\left(t_{1}^{\ell_{1}} t_{2}^{\ell_{2}}\right)^{\ell} \rrbracket= & \mathcal{A} \llbracket t_{1}^{\ell_{1}} \rrbracket ; \quad \mathcal{A} \llbracket t_{2}^{\ell_{2}} \rrbracket ; \\
& \quad \text { for each } \lambda x . t_{0}^{\ell_{0}} \text { in } \widehat{\mathrm{C}}\left(\ell_{1}\right) \text { do } \\
& \widehat{\mathrm{C}}\left[x \mapsto^{+} \widehat{\mathrm{C}}\left(\ell_{2}\right)\right] ; \quad \mathcal{A} \llbracket t_{0}^{\ell_{0}} \rrbracket ; \quad \widehat{\mathrm{C}}\left[\ell \mapsto^{+} \widehat{\mathrm{C}}\left(\ell_{0}\right)\right]
\end{aligned}
$$

The abstract evaluator $\mathcal{A} \llbracket \cdot \rrbracket$ is iterated until the finite cache reaches a fixed point. ${ }^{3}$ Since the cache size is polynomial in the program size, so is the running time, as the cache is monotonic - we put values in, but never take them out. Thus the analysis and any decision problems answered by the analysis are clearly computable within polynomial time.

An Example: Consider the following program, which we will return to discuss further in subsequent analyses:

$$
\left(\left(\lambda f \cdot\left(\left(f^{1} f^{2}\right)^{3}\left(\lambda y \cdot y^{4}\right)^{5}\right)^{6}\right)^{7}\left(\lambda x \cdot x^{8}\right)^{9}\right)^{10}
$$

The 0CFA is given by the following cache:

$$
\begin{array}{lll}
\widehat{\mathrm{C}}(1)=\{\lambda x\} & \widehat{\mathrm{C}}(6)=\{\lambda x, \lambda y\} & \\
\widehat{\mathrm{C}}(2)=\{\lambda x\} & \widehat{\mathrm{C}}(7)=\{\lambda f\} & \widehat{\mathrm{C}}(f)=\{\lambda x\} \\
\widehat{\mathrm{C}}(3)=\{\lambda x, \lambda y\} & \widehat{\mathrm{C}}(8)=\{\lambda x, \lambda y\} & \widehat{\mathrm{C}}(x)=\{\lambda x, \lambda y\} \\
\widehat{\mathrm{C}}(4)=\{\lambda y\} & \widehat{\mathrm{C}}(9)=\{\lambda x\} & \widehat{\mathrm{C}}(y)=\{\lambda y\} \\
\widehat{\mathrm{C}}(5)=\{\lambda y\} & \widehat{\mathrm{C}}(10)=\{\lambda x, \lambda y\} &
\end{array}
$$

where we write $\lambda x$ as shorthand for $\lambda x \cdot x^{8}$, etc.

\section{Henglein's simple closure analysis}

Simple closure analysis follows from an observation by Henglein some 15 years ago: he noted that the standard flow analysis can be computed in dramatically less time by changing the specification of flow constraints to use equality rather than containment [7]. The analysis bears a strong resemblance to simple typing: analysis can be performed by emitting a system of equality constraints and then solving them using unification, which can be computed in almost linear time with a union-find datastructure.

Consider a program with both $(f x)$ and $(f y)$ as subexpressions. Under 0CFA, whatever flows into $x$ and $y$ will also flow into the formal parameter of

${ }^{3}$ A single iteration of $\mathcal{A} \llbracket e \rrbracket$ may in turn make a recursive call $\mathcal{A} \llbracket e \rrbracket$ with no change in the cache, so care must be taken to avoid looping. This amounts to appealing to the coinductive hypothesis $\widehat{C} \models e$ in verifying $\widehat{C} \models e$. However, we consider this inessential detail, and it can safely be ignored for the purposes of obtaining our main results in which this behavior is never triggered. 
all abstractions flowing into $f$, but it is not necessarily true that whatever flows into $x$ also flows into $y$ and vice versa. However, under simple closure analysis, this is the case. For this reason, flows in simple closure analysis are said to be bidirectional.

The Analysis:

$$
\begin{aligned}
& \widehat{\mathrm{C}} \models x^{\ell} \text { iff } \widehat{\mathrm{C}}(x)=\widehat{\mathrm{C}}(\ell) \\
& \widehat{\mathrm{C}}=(\lambda x \cdot e)^{\ell} \text { iff } \lambda x \cdot e \in \widehat{\mathrm{C}}(\ell) \\
& \widehat{\mathrm{C}}=\left(t_{1}^{\ell_{1}} t_{2}^{\ell_{2}}\right)^{\ell} \text { iff } \widehat{\mathrm{C}}=t_{1}^{\ell_{1}} \wedge \widehat{\mathrm{C}}=t_{2}^{\ell_{2}} \wedge \forall \lambda x \cdot t_{0}^{\ell_{0}} \in \widehat{\mathrm{C}}\left(\ell_{1}\right): \\
& \quad \widehat{\mathrm{C}}=t_{0}^{\ell_{0}} \wedge \widehat{\mathrm{C}}\left(\ell_{2}\right)=\widehat{\mathrm{C}}(x) \wedge \widehat{\mathrm{C}}\left(\ell_{0}\right)=\widehat{\mathrm{C}}(\ell)
\end{aligned}
$$

The Algorithm: We write $\widehat{\mathrm{C}}\left[\ell \leftrightarrow \ell^{\prime}\right]$ to mean $\widehat{\mathrm{C}}\left[\ell \mapsto^{+} \widehat{\mathrm{C}}\left(\ell^{\prime}\right)\right]\left[\ell^{\prime} \mapsto^{+} \widehat{\mathrm{C}}(\ell)\right]$.

$$
\begin{aligned}
\mathcal{A} \llbracket x^{\ell} \rrbracket & =\widehat{\mathrm{C}}[\ell \leftrightarrow x] \\
\mathcal{A} \llbracket(\lambda x . e)^{\ell} \rrbracket= & \widehat{\mathrm{C}}\left[\ell \mapsto^{+}\{\lambda x . e\}\right] \\
\mathcal{A} \llbracket\left(t_{1}^{\ell_{1}} t_{2}^{\ell_{2}}\right)^{\ell} \rrbracket= & \mathcal{A} \llbracket t_{1}^{\ell_{1}} \rrbracket ; \mathcal{A} \llbracket t_{2}^{\ell_{2}} \rrbracket ; \\
& \text { for each } \lambda x . t_{0}^{\ell_{0}} \text { in } \widehat{\mathrm{C}}\left(\ell_{1}\right) \text { do } \\
& \widehat{\mathrm{C}}\left[x \leftrightarrow \ell_{2}\right] ; \quad \mathcal{A} \llbracket t_{0}^{\ell_{0}} \rrbracket ; \quad \widehat{\mathrm{C}}\left[\ell \leftrightarrow \ell_{0}\right]
\end{aligned}
$$

The abstract evaluator $\mathcal{A} \llbracket \cdot \rrbracket$ is iterated until a fixed point is reached. ${ }^{4}$ By similar reasoning to that given for $0 \mathrm{CFA}$, simple closure analysis is clearly computable within polynomial time.

An Example: Recall the example program of the previous section:

$$
\left(\left(\lambda f \cdot\left(\left(f^{1} f^{2}\right)^{3}\left(\lambda y \cdot y^{4}\right)^{5}\right)^{6}\right)^{7}\left(\lambda x \cdot x^{8}\right)^{9}\right)^{10}
$$

Notice that $\lambda x . x$ is applied to itself and then to $\lambda y . y$, so $x$ will be bound to both $\lambda x . x$ and $\lambda y . y$, which induces an equality between these two terms. Consequently, wherever 0CFA gives a flow set of $\{\lambda x\}$ or $\{\lambda y\}$, simple closure analysis will give $\{\lambda x, \lambda y\}$. The simple closure analysis is given by the following cache (new flows are underlined):

$$
\begin{array}{lll}
\widehat{\mathrm{C}}(1)=\{\lambda x, \lambda y\} & \widehat{\mathrm{C}}(6)=\{\lambda x, \lambda y\} & \\
\widehat{\mathrm{C}}(2)=\{\lambda x, \underline{\lambda y}\} & \widehat{\mathrm{C}}(7)=\{\lambda f\} & \widehat{\mathrm{C}}(f)=\{\lambda x, \lambda y\} \\
\widehat{\mathrm{C}}(3)=\{\lambda x, \overline{\lambda y}\} & \widehat{\mathrm{C}}(8)=\{\lambda x, \lambda y\} & \widehat{\mathrm{C}}(x)=\{\lambda x, \overline{\lambda y}\} \\
\widehat{\mathrm{C}}(4)=\{\lambda y, \underline{\lambda x}\} & \widehat{\mathrm{C}}(9)=\{\lambda x, \lambda y\} & \widehat{\mathrm{C}}(y)=\{\lambda y, \underline{\lambda x}\} \\
\widehat{\mathrm{C}}(5)=\{\lambda y, \underline{\lambda x}\} & \widehat{\mathrm{C}}(10)=\{\lambda x, \overline{\lambda y}\} &
\end{array}
$$

$\overline{4}$ The fine print of footnote 3 applies as well. 


\section{Linearity and normalization}

In this section, we show that when the program is linear every bound variable occurs exactly once-analysis and normalization are synonymous.

First, consider an evaluator for our language, $\mathcal{E} \llbracket \cdot \rrbracket$ :

$$
\begin{aligned}
& \mathcal{E} \llbracket \cdot \rrbracket: \mathbf{E x p} \rightarrow \mathbf{E n v} \rightarrow\langle\text { Term, Env }\rangle \\
& \mathcal{E} \llbracket x^{\ell} \rrbracket[x \mapsto c]= \\
& \mathcal{E} \llbracket(\lambda x . e)^{\ell} \rrbracket \rho=\langle\lambda x . e, \rho\rangle \\
& \mathcal{E} \llbracket\left(e_{1} e_{2}\right)^{\ell} \rrbracket \rho= \text { let }\left\langle\lambda x . e_{0}, \rho^{\prime}\right\rangle=\mathcal{E} \llbracket e_{1} \rrbracket \rho\left\lceil\mathbf{f v}\left(e_{1}\right)\right. \text { in } \\
& \text { let } c=\mathcal{E} \llbracket e_{2} \rrbracket \rho\left\lceil\mathbf{f v}\left(e_{2}\right)\right. \text { in } \\
& \mathcal{E} \llbracket e_{0} \rrbracket \rho^{\prime}[x \mapsto c]
\end{aligned}
$$

We use $\rho$ to range over environments, Env $=$ Var $\rightarrow\langle$ Term, Env $\rangle$, and let $c$ range over closures, each comprising a term and an environment that closes the term. The function $\mathbf{l a b}(\cdot)$ is extended to closures and environments by taking the union of all labels in the closure or in the range of the environment, respectively.

Notice that the evaluator "tightens" the environment in the case of an application, thus maintaining throughout evaluation that the domain of the environment is exactly the set of free variables in the expression. When evaluating a variable occurrence, there is only one mapping in the environment: the binding for this variable. Likewise, when constructing a closure, the environment does not need to be restricted: it already is.

In a linear program, each mapping in the environment corresponds to the single occurrence of a bound variable. So when evaluating an application, this tightening splits the environment $\rho$ into $\left(\rho_{1}, \rho_{2}\right)$, where $\rho_{1}$ closes the operator, $\rho_{2}$ closes the operand, and $\operatorname{dom}\left(\rho_{1}\right) \cap \operatorname{dom}\left(\rho_{2}\right)=\emptyset$.

Definition 1. Environment $\rho$ linearly closes $t$ (or $\langle t, \rho\rangle$ is a linear closure) iff $t$ is linear, $\rho$ closes $t$, and for all $x \in \operatorname{dom}(\rho), x$ occurs exactly once (free) in $t$, $\rho(x)$ is a linear closure, and for all $y \in \operatorname{dom}(\rho), x$ does not occur (free or bound) in $\rho(y)$. The size of a linear closure $\langle t, \rho\rangle$ is defined as:

$$
\begin{aligned}
|t, \rho| & =|t|+|\rho| \\
|x| & =1 \\
\left|\left(\lambda x \cdot t^{\ell}\right)\right| & =1+|t| \\
\left|\left(t_{1}^{\ell_{1}} t_{2}^{\ell_{2}}\right)\right| & =1+\left|t_{1}\right|+\left|t_{2}\right| \\
\left|\left[x_{1} \mapsto c_{1}, \ldots, x_{n} \mapsto c_{n}\right]\right| & =n+\sum_{i}\left|c_{i}\right|
\end{aligned}
$$

The following lemma states that evaluation of a linear closure cannot produce a larger value. This is the environment-based analog to the easy observation that $\beta$-reduction strictly decreases the size of a linear term.

Lemma 1. If $\rho$ linearly closes $t$ and $\mathcal{E} \llbracket t^{\ell} \rrbracket \rho=c$, then $|c| \leq|t, \rho|$. 
Proof. Straightforward by induction on $|t, \rho|$, reasoning by case analysis on $t$. Observe that the size strictly decreases in the application and variable case, and remains the same in the abstraction case.

Definition 2. A cache $\widehat{C}$ respects $\langle t, \rho\rangle$ (written $\widehat{C} \vdash t, \rho$ ) when,

1. $\rho$ linearly closes $t$,

2. $\forall x \in \operatorname{dom}(\rho) \cdot \rho(x)=\left\langle t^{\prime}, \rho^{\prime}\right\rangle \Rightarrow \widehat{\mathrm{C}}(x)=\left\{t^{\prime}\right\}$ and $\widehat{\mathrm{C}} \vdash t^{\prime}, \rho^{\prime}$, and

3. $\forall \ell \in \mathbf{l a b}(t) \backslash \mathbf{f v}(t), \widehat{\mathrm{C}}(\ell)=\emptyset$.

Clearly, $\emptyset \vdash t, \emptyset$ when $t$ is closed and linear, i.e. $t$ is a linear program.

Assume that the imperative algorithm $\mathcal{A} \llbracket \rrbracket$ of Section 3 is written in the obvious "cache-passing" functional style.

Theorem 1. If $\widehat{\mathrm{C}} \vdash t, \rho, \widehat{\mathrm{C}}(\ell)=\emptyset, \ell \notin \mathbf{l a b}(t, \rho), \mathcal{E} \llbracket t^{\ell} \rrbracket \rho=\left\langle t^{\prime}, \rho^{\prime}\right\rangle$, and $\mathcal{A} \llbracket t^{\ell} \rrbracket \widehat{\mathrm{C}}=$ $\widehat{\mathrm{C}}^{\prime}$, then $\widehat{\mathrm{C}}^{\prime}(\ell)=\left\{t^{\prime}\right\}, \widehat{\mathrm{C}}^{\prime} \vdash t^{\prime}, \rho^{\prime}$, and $\widehat{\mathrm{C}}^{\prime} \models t^{\ell}$.

An important consequence is noted in Corollary 1.

Proof. By induction on $|t, \rho|$, reasoning by case analysis on $t$.

- Case $t \equiv x$.

Since $\widehat{C} \vdash x, \rho$ and $\rho$ linearly closes $x$, thus $\rho=\left[x \mapsto\left\langle t^{\prime}, \rho^{\prime}\right\rangle\right]$ and $\rho^{\prime}$ linearly closes $t^{\prime}$. By definition,

$$
\begin{aligned}
\mathcal{E} \llbracket x^{\ell} \rrbracket \rho & =\left\langle t^{\prime}, \rho^{\prime}\right\rangle, \text { and } \\
\mathcal{A} \llbracket x^{\ell} \rrbracket \widehat{\mathrm{C}} & =\widehat{\mathrm{C}}[x \leftrightarrow \ell] .
\end{aligned}
$$

Again since $\widehat{\mathrm{C}} \vdash x, \rho, \widehat{\mathrm{C}}(x)=\left\{t^{\prime}\right\}$, with which the assumption $\widehat{\mathrm{C}}(\ell)=\emptyset$ implies

$$
\widehat{\mathrm{C}}[x \leftrightarrow \ell](x)=\widehat{\mathrm{C}}[x \leftrightarrow \ell](\ell)=\left\{t^{\prime}\right\},
$$

and therefore $\widehat{\mathrm{C}}[x \leftrightarrow \ell] \models x^{\ell}$. It remains to show that $\widehat{\mathrm{C}}[x \leftrightarrow \ell] \vdash t^{\prime}, \rho^{\prime}$. By definition, $\widehat{C} \vdash t^{\prime}, \rho^{\prime}$. Since $x$ and $\ell$ do not occur in $t^{\prime}, \rho^{\prime}$ by linearity and assumption, respectively, it follows that $\widehat{\mathrm{C}}[x \mapsto \ell] \vdash t^{\prime}, \rho^{\prime}$ and the case holds.

- Case $t \equiv \lambda x . e_{0}$.

By definition,

$$
\begin{aligned}
\mathcal{E} \llbracket\left(\lambda x . e_{0}\right)^{\ell} \rrbracket \rho & =\left\langle\lambda x . e_{0}, \rho\right\rangle, \\
\mathcal{A} \llbracket\left(\lambda x . e_{0}\right)^{\ell} \rrbracket \widehat{\mathrm{C}} & =\widehat{\mathrm{C}}\left[\ell \mapsto^{+}\left\{\lambda x . e_{0}\right\}\right],
\end{aligned}
$$

and by assumption $\widehat{C}(\ell)=\emptyset$, so $\widehat{C}\left[\ell \mapsto^{+}\left\{\lambda x . e_{0}\right\}\right](\ell)=\left\{\lambda x . e_{0}\right\}$ and therefore $\widehat{\mathrm{C}}\left[\ell \mapsto^{+}\left\{\lambda x . e_{0}\right\}\right] \models\left(\lambda x . e_{0}\right)^{\ell}$. By assumptions $\ell \notin \operatorname{lab}\left(\lambda x . e_{0}, \rho\right)$ and $\widehat{\mathrm{C}} \vdash$ $\lambda x . e_{0}, \rho$, it follows that $\widehat{\mathrm{C}}\left[\ell \mapsto^{+}\left\{\lambda x . e_{0}\right\}\right] \vdash \lambda x . e_{0}, \rho$ and the case holds. 
- Case $t \equiv t_{1}^{\ell_{1}} t_{2}^{\ell_{2}}$. Let

$$
\begin{aligned}
\mathcal{E} \llbracket t_{1} \rrbracket \rho\left\lceil\mathbf{f v}\left(t_{1}^{\ell_{1}}\right)\right. & =\left\langle v_{1}, \rho_{1}\right\rangle=\left\langle\lambda x \cdot t_{0}^{\ell_{0}}, \rho_{1}\right\rangle, \\
\mathcal{E} \llbracket t_{2} \rrbracket \rho\left\lceil\mathbf{f v}\left(t_{2}^{\ell_{2}}\right)\right. & =\left\langle v_{2}, \rho_{2}\right\rangle, \\
\mathcal{A} \llbracket t_{1} \rrbracket \widehat{\mathrm{C}} & =\widehat{\mathrm{C}}_{1}, \text { and } \\
\mathcal{A} \llbracket t_{2} \rrbracket \widehat{\mathrm{C}} & =\widehat{\mathrm{C}}_{2} .
\end{aligned}
$$

Clearly, for $i \in\{1,2\}, \widehat{\mathrm{C}} \vdash t_{i}, \rho\left\lceil\mathbf{f v}\left(t_{i}\right)\right.$ and

$$
1+\sum_{i} \mid t_{i}^{\ell_{i}}, \rho\left\lceil\mathbf{f v}\left(t_{i}^{\ell_{i}}\right)|=|\left(t_{1}^{\ell_{1}} t_{2}^{\ell_{2}}\right), \rho \mid .\right.
$$

By induction, for $i \in\{1,2\}: \widehat{\mathrm{C}}_{i}\left(\ell_{i}\right)=\left\{v_{i}\right\}, \widehat{\mathrm{C}}_{i} \vdash\left\langle v_{i}, \rho_{i}\right\rangle$, and $\widehat{\mathrm{C}}_{i}=t_{i}^{\ell_{i}}$. From this, it is straightforward to observe that $\widehat{C}_{1}=\widehat{C} \cup \widehat{\mathrm{C}}_{1}^{\prime}$ and $\widehat{\mathrm{C}}_{2}=\widehat{\mathrm{C}} \cup \widehat{\mathrm{C}}_{2}^{\prime}$ where $\widehat{C}_{1}^{\prime}$ and $\widehat{C}_{2}^{\prime}$ are disjoint. So let $\widehat{C}_{3}=\left(\widehat{C}_{1} \cup \widehat{C}_{2}\right)\left[x \leftrightarrow \ell_{2}\right]$. It is clear that $\widehat{\mathrm{C}}_{3}=t_{i}^{\ell_{i}}$. Furthermore,

$$
\begin{aligned}
\widehat{\mathrm{C}}_{3} & \vdash t_{0}, \rho_{1}\left[x \mapsto\left\langle v_{2}, \rho_{2}\right\rangle\right], \\
\widehat{\mathrm{C}}_{3}\left(\ell_{0}\right) & =\emptyset, \text { and } \\
\ell_{0} & \notin \operatorname{lab}\left(t_{0}, \rho_{1}\left[x \mapsto\left\langle v_{2}, \rho_{2}\right\rangle\right]\right) .
\end{aligned}
$$

By Lemma $1,\left|v_{i}, \rho_{i}\right| \leq \mid t_{i}, \rho\left\lceil\mathbf{f v}\left(t_{i}\right) \mid\right.$, therefore

$$
\left|t_{0}, \rho_{1}\left[x \mapsto\left\langle v_{2}, \rho_{2}\right\rangle\right]\right|<\left|\left(t_{1}^{\ell_{1}} t_{2}^{\ell_{2}}\right)\right| .
$$

Let

$$
\begin{aligned}
\mathcal{E} \llbracket t_{0}^{\ell_{0}} \rrbracket \rho_{1}\left[x \mapsto\left\langle v_{2}, \rho_{2}\right\rangle\right] & =\left\langle v^{\prime}, \rho^{\prime}\right\rangle, \\
\mathcal{A} \llbracket t_{0}^{\ell_{0}} \rrbracket \widehat{\mathrm{C}}_{3} & =\widehat{\mathrm{C}}_{4},
\end{aligned}
$$

and by induction, $\widehat{\mathrm{C}}_{4}\left(\ell_{0}\right)=\left\{v^{\prime}\right\}, \widehat{\mathrm{C}}_{4} \vdash v^{\prime}, \rho^{\prime}$, and $\widehat{\mathrm{C}}_{4} \models v^{\prime}$. Finally, observe that $\widehat{\mathrm{C}}_{4}\left[\ell \leftrightarrow \ell_{0}\right](\ell)=\widehat{\mathrm{C}}_{4}\left[\ell \leftrightarrow \ell_{0}\right]\left(\ell_{0}\right)=\left\{v^{\prime}\right\}, \widehat{\mathrm{C}}_{4}\left[\ell \leftrightarrow \ell_{0}\right] \vdash v^{\prime}, \rho^{\prime}$, and $\widehat{\mathrm{C}}_{4}[\ell \leftrightarrow$ $\left.\ell_{0}\right]=\left(t_{1}^{\ell_{1}} t_{2}^{\ell_{2}}\right)^{\ell}$, so the case holds.

We can now establish the correspondence between analysis and evaluation.

Corollary 1. If $\widehat{C}$ is the simple closure analysis of a linear program $t^{\ell}$, then $\mathcal{E} \llbracket t^{\ell} \rrbracket \emptyset=\left\langle v, \rho^{\prime}\right\rangle$ where $\widehat{\mathrm{C}}(\ell)=\{v\}$ and $\widehat{\mathrm{C}} \vdash v, \rho^{\prime}$.

By a simple replaying of the proof substituting the containment constraints of 0CFA for the equality constraints of simple closure analysis, it is clear that the same correspondence can be established, and therefore 0CFA and simple closure analysis are identical for linear programs.

Corollary 2. If $e$ is a linear program, then $\widehat{\mathrm{C}}$ is the simple closure analysis of $e$ iff $\widehat{\mathrm{C}}$ is the 0CFA of $e$. 
Discussion: Returning to our earlier question of the computationally potent ingredients in a static analysis, we can now see that when the term is linear, whether flows are directional and bidirectional is irrelevant. For these terms, simple closure analysis, 0CFA, and evaluation are equivalent. And, as we will see, when an analysis is exact for linear terms, the analysis will have a PTIMEhardness bound.

\section{Lower bounds for flow analysis}

There are at least two fundamental ways to reduce the complexity of analysis. One is to compute more approximate answers, the other is to analyze a syntactically restricted language.

We use linearity as the key ingredient in proving lower bounds on analysis. This shows not only that simple closure analysis and other flow analyses are PTIME-complete, but the result is rather robust in the face of analysis design based on syntactic restrictions. This is because we are able to prove the lower bound via a highly restricted programming language - the linear $\lambda$-calculus. So long as the subject language of an analysis includes the linear $\lambda$-calculus, and is exact for this subset, the analysis must be at least PTIME-hard.

The decision problem answered by flow analysis, described colloquially in Section 1, is formulated as follows:

Flow Analysis Problem: Given a closed expression $e$, a term $v$, and label $\ell$, is $v \in \widehat{\mathrm{C}}(\ell)$ in the analysis of $e$ ?

Theorem 2. If analysis corresponds to evaluation on linear terms, the analysis is PTIME-hard.

The proof is by reduction from the canonical PTIME-complete problem [15]:

Circuit Value Problem: Given a Boolean circuit $C$ of $n$ inputs and one output, and truth values $\boldsymbol{x}=x_{1}, \ldots, x_{n}$, is $\boldsymbol{x}$ accepted by $C$ ?

An instance of the circuit value problem can be compiled, using only logarithmic space, into an instance of the flow analysis problem following the construction in [9]. Briefly, the circuit and its inputs are compiled into a linear $\lambda$-term, which simulates $C$ on $\boldsymbol{x}$ via evaluation - it normalizes to true if $C$ accepts $\boldsymbol{x}$ and false otherwise. But since the analysis faithfully captures evaluation of linear terms, and our encoding is linear, the circuit can be simulated by flow analysis.

The encodings work like this: $t t$ is the identity on pairs, and ff is the swap. Boolean values are either $\langle t t, f f\rangle$ or $\langle f f, t t\rangle$, where the first component is the "real" value, and the second component is the complement.

$$
\begin{aligned}
t t \equiv \lambda p . \text { let }\langle x, y\rangle & =p \text { in }\langle x, y\rangle & \text { True } \equiv\langle t t, f f\rangle \\
f f \equiv \lambda p . \text { let }\langle x, y\rangle & =p \text { in }\langle y, x\rangle & \text { False } \equiv\langle f f, t t\rangle
\end{aligned}
$$

The simplest connective is Not, which is an inversion on pairs, like ff. A linear copy connective is defined as:

$$
\text { Copy } \equiv \lambda b \text {.let }\langle u, v\rangle=b \text { in }\langle u\langle t t, f f\rangle, v\langle f f, t t\rangle\rangle .
$$


The coding is easily explained: suppose $b$ is True, then $u$ is identity and $v$ twists; so we get the pair $\langle$ True, True $\rangle$. Suppose $b$ is False, then $u$ twists and $v$ is identity; we get $\langle$ False, False $\rangle$.

The And connective is defined as:

$$
\begin{aligned}
& \text { And } \equiv \lambda b_{1} \cdot \lambda b_{2} . \\
& \text { let }\left\langle u_{1}, v_{1}\right\rangle=b_{1} \text { in } \\
& \text { let }\left\langle u_{2}, v_{2}\right\rangle=b_{2} \text { in } \\
& \text { let }\left\langle p_{1}, p_{2}\right\rangle=u_{1}\left\langle u_{2}, f f\right\rangle \text { in } \\
& \text { let }\left\langle q_{1}, q_{2}\right\rangle=v_{1}\left\langle t t, v_{2}\right\rangle \text { in } \\
&\left\langle p_{1}, q_{1} \circ p_{2} \circ q_{2} \circ f f\right\rangle .
\end{aligned}
$$

Conjunction works by computing pairs $\left\langle p_{1}, p_{2}\right\rangle$ and $\left\langle q_{1}, q_{2}\right\rangle$. The former is the usual conjuction on the first components of the Booleans $b_{1}, b_{2}: u_{1}\left\langle u_{2}, f f\right\rangle$ can be read as "if $u_{1}$ then $u_{2}$, otherwise false (ff)." The latter is (exploiting deMorgan duality) the disjunction of the complement components of the Booleans: $v_{1}\left\langle t t, v_{2}\right\rangle$ is read as "if $v_{1}$ (i.e. if not $u_{1}$ ) then true $(t t)$, otherwise $v_{2}$ (i.e. not $u_{2}$ )." The result of the computation is equal to $\left\langle p_{1}, q_{1}\right\rangle$, but this leaves $p_{2}, q_{2}$ unused, which would violate linearity. However, there is symmetry to this garbage, which allows for its disposal. Notice that, while we do not know whether $p_{2}$ is $t t$ or $f f$ and similarly for $q_{2}$, we do know that one of them is tt while the other is ff. Composing the two together, we are guaranteed that $p_{2} \circ q_{2}=f f$. Composing this again with another twist $(f f)$ results in the identity function $p_{2} \circ q_{2} \circ f f=t t$. Finally, composing this with $q_{1}$ is just equal to $q_{1}$, so $\left\langle p_{1}, q_{1} \circ p_{2} \circ q_{2} \circ f f\right\rangle=\left\langle p_{1}, q_{1}\right\rangle$, which is the desired result, but the symmetric garbage has been annihilated, maintaining linearity.

This hacking, with its self-annihilating garbage, is an improvement over that given in [16] and allows Boolean computation without K-redexes, making the lower bound stronger, but also preserving all flows. In addition, it is the best way to do circuit computation in multiplicative linear logic, and is how you compute similarly in non-affine typed $\lambda$-calculus.

We know from Corollary 1 that normalization and analysis of linear programs are synonymous, and our encoding of circuits will faithfully simulate a given circuit on its inputs, evaluating to true iff the circuit accepts its inputs. But it does not immediately follow that the circuit value problem can be reduced to the flow analysis problem. Let $\|C, \boldsymbol{x}\|$ be the encoding of the circuit and its inputs. It is tempting to think the instance of the flow analysis problem could be stated:

is True in $\widehat{\mathrm{C}}(\ell)$ in the analysis of $\|C, \boldsymbol{x}\|^{\ell}$ ?

The problem with this is there may be many syntactic instances of "True." Since the flow analysis problem must ask about a particular one, this reduction will not work. The fix is to use a context which expects a boolean expression and induces a particular flow (that can be asked about in the flow analysis problem) iff that expression evaluates to a true value [9].

Corollary 3. Simple closure analysis is PTIME-complete. 


\section{Other monovariant analyses}

In this section, we survey some of the existing monovariant analyses that either approximate or restrict 0CFA to obtain faster analysis times. In each case, we sketch why these analyses are complete for PTIME.

\subsection{Ashley and Dybvig's sub-0CFA}

In [8], Ashley and Dybvig develop a general framework for specifying and computing flow analyses, which can be instantiated to obtain 0CFA or Jagannathan and Weeks' polynomial 1CFA [17], for example. They also develop a class of instantiations of their framework dubbed sub-OCFA that is faster to compute, but less accurate than 0CFA.

This analysis works by explicitly bounding the number of times the cache can be updated for any given program point. After this threshold has been crossed, the cache is updated with a distinguished unknown value that represents all possible $\lambda$-abstractions in the program. Bounding the number of updates to the cache for any given location effectively bounds the number of passes over the program an analyzer must make, producing an analysis that is $O(n)$ in the size of the program. Empirically, Ashley and Dybvig observe that setting the bound to 1 yields an inexpensive analysis with no significant difference in enabling optimizations with respect to 0CFA.

The idea is the cache gets updated once ( $n$ times in general) before giving up and saying all $\lambda$-abstractions flow out of this point. But for a linear term, the cache is only updated at most once for each program point. Thus we conclude even when the sub-0CFA bound is 1, the problem is PTIME-complete.

As Ashley and Dybvig note, for any given program, there exists an analysis in the sub-0CFA class that is identical to 0CFA (namely by setting $n$ to the number of passes 0CFA makes over the given program). We can further clarify this relationship by noting that for all linear programs, all analyses in the sub0CFA class are identical to 0CFA (and thus simple closure analysis).

\subsection{Subtransitive 0CFA}

Heintze and McAllester [6] have shown that the "cubic bottleneck" of computing full 0CFA - that is, computing all the flows in a program - cannot be avoided in general without combinatorial breakthroughs: the problem is 2NPDA-hard, for which the "the cubic time decision procedure [...] has not been improved since its discovery in 1968."

Given the unlikeliness of improving the situation in general, Heintze and McAllester [10] identify several simpler flow questions (including the decision problem discussed in the paper, which is the simplest; answers to any of the other questions imply an answer to this problem). They give algorithms for simply typed terms that answer these restricted flow problems, which under certain conditions, compute in less than cubic time. 
Their analysis is linear with respect to a program's graph, which in turn, is bounded by the size of the program's type. Thus, bounding the size of a program's type results in a linear bound on the running times of these algorithms. If this type bound is removed, though, it is clear that even these simplified flow problems (and their bidirectional-flow analogs), are complete for PTIME: observe that every linear term is simply typable, however in our lower bound construction, the type size is proportional to the size of the circuit being simulated. As they point out, when type size is not bounded, the flow graph may be exponentially larger than the program, in which case the standard cubic algorithm is preferred.

Independently, Mossin [18] developed a type-based analysis that, under the assumption of a constant bound on the size of a program's type, can answer restricted flow questions such as single source/use in linear time with respect to the size of the explicitly typed program. But again, removing this imposed bound results in PTIME-completeness.

As Hankin et al. [19] point out: both Heintze and McAllester's and Mossin's algorithms operate on type structure (or structure isomorphic to type structure), but with either implicit or explicit $\eta$-expansion. For simply typed terms, this can result in an exponential blow-up in type size. It is not surprising then, that given a much richer graph structure, the analysis can be computed quickly. In this light, recent results on 0CFA of $\eta$-expanded, simply typed programs can be seen as an improvement of the subtransitive flow analysis since it works equally well for languages with first-class control and can be performed with only a fixed number of pointers into the program structure, i.e. it is computable in LOGSPACE (and in other words, PTIME $=$ LOGSPACE up to $\eta$ ) [9].

\section{Conclusions and perspective}

When an analysis is exact, it will be possible to establish a correspondence with evaluation. The richer the language for which analysis is exact, the harder it will be to compute the analysis. As an example in the extreme, Mossin [20] developed a flow analysis that is exact for simply typed terms. The computational resources that may be expended to compute this analysis are ipso facto not bounded by any elementary recursive function [21]. However, most flow analyses do not approach this kind of expressivity. By way of comparison, 0CFA only captures PTIME, and yet researchers have still expending a great deal of effort deriving approximations to 0CFA that are faster to compute. But as we have shown for a number of them, they all coincide on linear terms, and so they too capture PTIME.

We should be clear about what is being said, and not said. There is a considerable difference in practice between linear algorithms (nominally considered efficient) and cubic algorithms (still feasible, but taxing for large inputs), even though both are polynomial-time. PTIME-completeness does not distinguish the two. But if a sub-polynomial (e.g., LOGSPACE) algorithm was found for this sort of flow analysis, it would depend on (or lead to) things we do not know $($ LOGSPACE $=$ PTIME $)$. Likewise, were a parallel implementation of this flow 
analysis to run in logarithmic time (i.e., NC), we would consequently be able to parallelize every polynomial time algorithm similarly.

A fundamental question we need to be able to answer is this: what can be deduced about a long-running program with a time-bounded analyzer? When we statically analyze exponential-time programs with a polynomial-time method, there should be a analytic bound on what we can learn at compile-time: a theorem delineating how exponential time is being viewed through the compressed, myopic lens of polynomial time computation.

For example, a theorem due to Statman [21] says this: let $\mathbf{P}$ be a property of simply-typed $\lambda$-terms that we would like to detect by static analysis, where $\mathbf{P}$ is invariant under reduction (normalization), and is computable in elementary time (polynomial, or exponential, or doubly-exponential, or...). Then $\mathbf{P}$ is a trivial property: for any type $\tau, \mathbf{P}$ is satisfied by all or none of the programs of type $\tau$. Henglein and Mairson [22] have complemented these results, showing that if a property is invariant under $\beta$-reduction for a class of programs that can encode all Turing Machines solving problems of complexity class F using reductions from complexity class G, then any superset is either F-complete or trivial. Simple typability has this property for linear and linear affine $\lambda$-terms [22, 16], and these terms are sufficient to code all polynomial-time Turing Machines.

We would like to prove some analogs of these theorems, with or without the typing condition, but weakening the condition of "invariant under reduction" to some approximation analogous to the approximations of flow analysis, as described above. We are motivated as well by yardsticks such as Shannon's theorem from information theory [23]: specify a bandwidth for communication and an error rate, and Shannon's results give bounds on the channel capacity. We too have essential measures: the time complexity of our analysis, the asymptotic differential between that bound and the time bound of the program we are analyzing. There ought to be a fundamental result about what information can be yielded as a function of that differential. At one end, if the program and analyzer take the same time, the analyzer can just run the program to find out everything. At the other end, if the analyzer does no work (or a constant amount of work), nothing can be learned. Analytically speaking, what is in between?

Acknowledgments: We are grateful to Olin Shivers and Matt Might for a long, fruitful, and ongoing dialogue on flow analysis. We thank the anonymous reviewers for insightful comments. The first author also thanks the researchers of the Northeastern University Programming Research Lab for the hospitality and engaging discussions had as a visiting lecturer over the last year.

\section{References}

1. Jones, N.D.: Flow analysis of lambda expressions (preliminary version). In: Proceedings of the 8th Colloquium on Automata, Languages and Programming, London, UK, Springer-Verlag (1981) 114-128

2. Sestoft, P.: Replacing function parameters by global variables. Master's thesis, DIKU, University of Copenhagen, Denmark (October 1988) Master's thesis no. 254. 
3. Shivers, O.: Control-Flow Analysis of Higher-Order Languages, or Taming Lambda. PhD thesis, School of Computer Science, Carnegie Mellon University, Pittsburgh, Pennsylvania (May 1991) Technical Report CMU-CS-91-145.

4. Midtgaard, J.: Control-flow analysis of functional programs. Technical Report BRICS RS-07-18, DAIMI, Department of Computer Science, University of Aarhus, Aarhus, Denmark (December 2007)

5. Shivers, O.: Control flow analysis in Scheme. In: PLDI '88: Proceedings of the ACM SIGPLAN 1988 conference on Programming Language design and Implementation, ACM (1988) 164-174

6. Heintze, N., McAllester, D.: On the cubic bottleneck in subtyping and flow analysis. In: LICS '97: Proceedings of the 12th Annual IEEE Symposium on Logic in Computer Science, Washington, DC, USA, IEEE Computer Society (1997) 342

7. Henglein, F.: Simple closure analysis. DIKU Semantics Report D-193 (1992)

8. Ashley, J.M., Dybvig, R.K.: A practical and flexible flow analysis for higher-order languages. ACM Trans. Program. Lang. Syst. 20(4) (1998) 845-868

9. Van Horn, D., Mairson, H.G.: Relating complexity and precision in control flow analysis. In: Proceedings of the 2007 ACM SIGPLAN International Conference on Functional Programming, ACM Press (2007) 85-96

10. Heintze, N., McAllester, D.: Linear-time subtransitive control flow analysis. In: PLDI '97: Proceedings of the ACM SIGPLAN 1997 conference on Programming language design and implementation, ACM (1997) 261-272

11. Girard, J.Y.: Linear logic: its syntax and semantics. In: Proceedings of the workshop on Advances in linear logic, Cambridge University Press (1995)

12. Sestoft, P.: Replacing function parameters by global variables. In: FPCA '89: Proceedings of the fourth international conference on Functional programming languages and computer architecture, ACM (1989) 39-53

13. Mossin, C.: Flow Analysis of Typed Higher-Order Programs. PhD thesis, DIKU, University of Copenhagen (January (revised August) 1997)

14. Nielson, F., Nielson, H.R., Hankin, C.: Principles of Program Analysis. SpringerVerlag New York, Inc., Secaucus, NJ, USA (1999)

15. Ladner, R.E.: The circuit value problem is log space complete for $P$. SIGACT News 7(1) (1975) 18-20

16. Mairson, H.G.: Linear lambda calculus and PTIME-completeness. Journal of Functional Programming 14(6) (2004) 623-633

17. Jagannathan, S., Weeks, S.: A unified treatment of flow analysis in higher-order languages. In: Proceedings of the 22nd ACM SIGPLAN-SIGACT Symposium on Principles of Programming Languages, ACM (1995) 393-407

18. Mossin, C.: Higher-order value flow graphs. Nordic J. of Computing 5(3) (1998) $214-234$

19. Hankin, C., Nagarajan, R., Sampath, P.: Flow analysis: games and nets. In: The essence of computation: complexity, analysis, transformation. Springer-Verlag New York, Inc. (2002) 135-156

20. Mossin, C.: Exact flow analysis. In: SAS '97: Proceedings of the 4th International Symposium on Static Analysis, London, UK, Springer-Verlag (1997) 250-264

21. Statman, R.: The typed $\lambda$-calculus is not elementary recursive. Theor. Comput. Sci. 9 (1979) 73-81

22. Henglein, F., Mairson, H.G.: The complexity of type inference for higher-order lambda calculi. In: POPL '91: Proceedings of the 18th ACM SIGPLAN-SIGACT Symposium on Principles of Programming Languages, ACM (1991) 119-130

23. Shannon, C.E.: A mathematical theory of communication. Bell System Technical Journal 27 (1948) 